\title{
Regional gray matter volume mediates the relationship between family socioeconomic status and depression-related trait in a young healthy sample
}

\author{
Junyi Yang ${ }^{1,2}$ - Huijuan Liu ${ }^{1,2}$ • Dongtao Wei ${ }^{1,2}$ - Wei Liu ${ }^{1,2}$. Jie Meng ${ }^{1,2}$. \\ Kangcheng Wang $^{1,2}$ - Lei Hao ${ }^{1,2}$. Jiang Qiu ${ }^{1,2}$
}

Published online: 5 August 2015

(C) Psychonomic Society, Inc. 2015

\begin{abstract}
Socioeconomic status (SES) is a complex phenomenon that is predicted by a broad spectrum of variables and is often conceptualized as a combination of financial, occupational, and educational influences. SES is associated with symptoms of depression and depression-related personality traits. Many studies have focused on the relationship between SES and depression symptoms in clinical subjects; however, there are few studies of the neural basis of the relationship between SES and depression-related personality traits in nonclinical subjects. Thus, in the present study, we studied the neural basis of the relationship between SES and depressionrelated traits. The first step was to use voxel-based morphometry (VBM) to investigate the neuroanatomical basis underlying family SES in a large sample of healthy subjects. We found a significant negative correlation between family SES and the medial prefrontal cortex (mPFC) that extending to the anterior cingulate cortex (ACC). Then, the mediation analysis revealed that the correlation between family SES, especially family income, and depression-related traits were mediated by the region of the $\mathrm{mPFC}$ that extending to the ACC volume. These findings suggest that the family income may play more important role in driving depression-related traits than that of parental education. They also suggest that the mPFC extension to the ACC may play an important role in the correlation between familial SES, especially with respect to family income and the depression-related traits.
\end{abstract}

Jiang Qiu

qiuj318@swu.edu.cn

1 Key Laboratory of Cognition and Personality (SWU), Ministry of Education, Chongqing 400715, China

2 Department of Psychology, Southwest University, Chongqing 400715, China
Keywords Socioeconomic status · Voxel-based morphometry $\cdot$ Depression-related traits $\cdot$ Medial prefrontal cortex $\cdot$ Anterior cingulate cortex

\section{Introduction}

The socioeconomic status (SES) and education, two important environmental variables, are used as predictors of people's health outcomes, mortality, social skills and cognitive abilities (Brito \& Noble, 2014; Marioni et al., 2014). SES not only plays a role in epidemiological parlance that could be partially genetic in origin but also has a potentially important role as a moderator of genetic influences on intelligence(Marioni et al., 2014). SES is a complex phenomenon and is a multidimensional construct, combining objective factors such as an individual's (or parent's) education, occupation, and income (Adler et al., 1994; Dubow, Huesmann, Boxer, Pulkkinen, \& Kokko, 2006). Higher SES families can afford to provide their children an array of services, goods, parental actions, and social connections that potentially benefit them. Many lower SES children lack access to the same resources and experiences, thus putting them at risk for developmental problems, such as worse physical and psychological health and impaired cognitive and emotional development (Bradley \& Corwyn, 2002; Brooks-Gunn \& Duncan, 1997; Hackman, Farah, \& Meaney, 2010; McLoyd, 1998). Growing evidence suggests that exposure to lower SES could increase the risk for mental illnesses, such as depression and anxiety disorders, throughout life (Adler et al., 1994; Marmot, Kogevinas, \& Elston, 1987). SES-related mental health disparities have become an increasingly important and visible part of the public health landscape (Zimmerman \& Katon, 2005). For example, many researchers have focused on the relationship of SES disparities with depression, and their work indicates that SES is associated with 
depressive symptoms and a greater likelihood of depression (Goodman, 1999; Goodman, Slap, \& Huang, 2003; McLoyd, 1997; Tracy, Zimmerman, Galea, McCauley, \& Stoep, 2008).

Neuroticism, a personality dimension characterized by a general tendency to experience negative emotion, has been suggested to be a predisposing factor to clinical depression (Flett, Hewitt, Endler, \& Bagby, 1995; Sen et al., 2003). It is also associated with the severity of patients' current depressive symptoms and with the vulnerability to depression within nonclinical groups (Saklofske, Kelly, \& Janzen, 1995). The depression-related trait of the neuroticism scale (N3) was used to measure normal individual differences in the tendency to experience a depressive affect (Huprich, 2000), and it has been positively associated with depressive symptoms (Chioqueta \& Stiles, 2005). Contrary to the vast amount of neuroimaging studies on SES and depression symptoms in clinical subjects, few studies have examined the neural basis of the relationship between SES and depression-related personality traits in nonclinical subjects. Thus, in present study we focus on the neural basis of the relationship between SES and depression-related traits.

With the development of cognitive neuroscience, more and more researchers have focused on the relationship between SES and the development of brain structures. For example, Gianaros and his colleagues found that subjective social status was significantly associated with reduced gray matter volume in the perigenual area of the anterior cingulate cortex (pACC), which is involved in experiencing emotions and regulating behavioral and physiological reactivity to stress (Gianaros et al., 2007). In addition, some researchers have found significant positive correlations between family income and hippocampal size, with children and adolescents from lower SES families having smaller hippocampal volumes (Hanson, Chandra, Wolfe, \& Pollak, 2011; Luby et al., 2013; Noble, Houston, Kan, \& Sowell, 2012) and smaller amygdala volumes (Luby et al., 2013). Moreover, educational studies indicate that there is a significant association between right hippocampal, amygdala volumes and paternal education levels (Hanson et al., 2011; Robert \& House, 2000). A recent study examined a sample of 283 healthy children to investigate the relationship between SES and prefrontal cortical thickness, and they found that parental education significantly predicted cortical thickness in the right anterior cingulate gyrus and left superior frontal gyrus (Lawson, Duda, Avants, Wu, \& Farah, 2013). In summary, SES is associated with the development of brain structures that play an important role in emotion, such as the hippocampus, anterior cingulate cortex, and amygdala.

Interindividual differences are not limited to explaining the neural basis of performance differences between individual participants, but they can be used to reveal the neural circuitry associated with a particular cognitive function (Kanai \& Rees,
2011). There has been a rapid increase in the number of studies examining interindividual differences in human behavior and their association with structural features of the brain (Fleming, Weil, Nagy, Dolan, \& Rees, 2010; Tomassini et al., 2011; Washburn, Smith, \& Taglialatela, 2005). Measures of brain structures can be viewed as mediators between SES and cognition or as outcome variables in their own right (Brito \& Noble, 2014). In the current study, we investigated the neural bias of family SES and depression-related traits from the perspective of interindividual differences in healthy subjects. First, we used voxel-based morphometry (VBM) on structural magnetic resonance images to identify the relationship between family SES and the volume of brain regions on a whole-brain level. Second, we used mediation analysis to examine whether the specific regional gray matter volume (rGMV) mediated the relationship between depression-related traits and family SES. Based on the previous neuroscience findings on SES (Gianaros et al., 2008; Otero, 1997; Raizada, Richards, Meltzoff, \& Kuhl, 2008; Tomarken, Dichter, Garber, \& Simien, 2004), we hypothesized that family SES might affect the rGMV of prefrontal cortex (PFC) regions linked to cognitive-emotional functions (Kim \& Hamann, 2007; Lane et al., 1998; Phan et al., 2004), as well as ACC, hippocampus or amygdala, which play important roles in regulation of cognitive and emotional behavior (Bush, Luu, \& Posner, 2000; Devinsky, Morrell, \& Vogt, 1995; Posner, 1995). In addition, we hypothesized that there are some rGMV, and especially the regions related to family SES would mediate the relationship between depressionrelated traits and family SES.

\section{Method}

\section{Participants}

A total of 253 right-handed, healthy volunteers (150 women and 103 men; mean age $=19.96$ years, $S D=1.35$ years, range 18-27 years) participated in the study as part of our ongoing project to examine the associations between brain imaging, creativity, and mental health. All participants were university students or graduated students from the local community of Southwest University, China. Participants were screened for past history of mental illnesses and neurological disorders by a self-report questionnaire before the scanning. The participants who had a history of psychiatric or neurological disorders, had received mental health treatment, or had taken psychiatric medications were excluded. All participants provided written informed consent prior to the study. The Brain Imaging Center Institutional Review Board of Southwest China University approved this study and the experiment procedure, which was in accordance with the standards of the Declaration of Helsinki (1991). 


\section{Study measures}

\section{Family annual income}

Data were collected using discrete variables: (1) annual income < RMB 5,000; (2) annual income RMB 5,000-15000; (3) annual income RMB 15,001-30,000; (4) annual income RMB 30,001-50,000; (5) annual income RMB 50,001-100, 000 ; (6) annual income $>$ RMB 100,000. The values $1-6$ were used in subsequent regression analyzes (Takeuchi et al., 2014).

\section{The educational qualification of both parents}

There were five options: (1) elementary school graduate or below; (2) junior high school graduate; (3) high school graduate; (4) university graduate; (5) above university graduate; and each choice was converted into the number of years according to the Chinese education system: (1) 6 years; (2) 9 years; (3) 12 years; (4) 16 years; (5) 19 years. The average of the converted values for each parent was used in the analyses (Takeuchi et al., 2014).

\section{Family socioeconomic status (SES)}

We computed a composite indicator of family SES by averaging the standardized ( $z$ score) values of the two index variables: educational qualifications and family annual income for each participant (Gianaros et al., 2007; Manuck et al., 2005; K. A. Matthews, Flory, Muldoon, \& Manuck, 2000).

\section{Parental subjective social status rating by participants}

Ratings of subjective social status were derived from the MacArthur Scale of Subjective Social Status (Adler, Epel, Castellazzo, \& Ickovics, 2000; Gianaros et al., 2007). For this 9-point scale, participants were provided with an image of a "social ladder" and were asked to place an check mark on the rung corresponding to their parental standing or ranking in relation to other individuals in China according to income, education, and occupational prestige. Previous studies have suggested the measure of the subjective SES has adequate test-retest reliability $(r=.62, p<.01$; Operario, Adler, \& Williams, 2004). The average of the converted values for each parent was used in the analyses.

\section{Measuring the level of depression-related personality traits}

Family SES affects personality development and significantly associated with the big five personality traits (Cheng \& Furnham, 2014). In the study, personality traits were assessed using the revised Neuroticism-Extraversion-Openness Personality Inventory (NEO-PI-R; Costa \& McCrae, 1992b).
The NEO-PI-R consists of 240 items and is based on a fivefactor model of personality. All these factors are divided into six subscales. The subscales of neuroticism are Anxiety, Hostility, Depression, Self-Consciousness, Impulsiveness and Vulnerability (D. Wei et al., 2014). Previous studies have shown that the NEO-PI-R has good reliability and validity(Costa \& McCrae, 1992a; McCrae, 2011). In our study, we mainly focused on depression-related traits of the neuroticism (N3) dimension, based on the a priori hypothesis predicting a relationship between depression-related traits and family SES (Goodman et al., 2003; Tracy et al., 2008). The N3 consists of eight items, including two reverse-scored items to reduce the effects of acquiescence. The items are answered on a 5-point Likert scale, ranging from strongly disagree to strongly agree. The $\mathrm{N} 3$ is usually used as a measure of depression-related traits (Bienvenu et al., 2004; Terracciano et al., 2010; Vossen, van Os, Hermens, \& Lousberg, 2006).

\section{Assessment of general intelligence}

To examine intellectual ability, participants completed the Combined Raven's Test (CRT), which is a recognized intelligence test with a high degree of reliability and validity (Tang et al., 2012). The reliability coefficient was 0.92 (Ming, 1989). The CRT included the Raven's standard progressive matrix (C, D, E sets) and Raven's colored progressive matrix (A, $\mathrm{AB}, \mathrm{B}$ sets), consisting of 72 items revised by the Psychology Department of East China Normal University in 1989. The score of this test (the number of correct answers given in 40 minutes) was used as a psychometric index of individual intelligence. In line with standard practice, the current study focused on the total score of the test (Jaeggi, Buschkuehl, Jonides, \& Perrig, 2008; Takeuchi et al., 2011).

\section{MRI data acquisition}

A 3.0-T Siemens Trio MRI scanner (Siemens Medical, Erlangen, Germany) was used to obtain MR images. A magnetization-prepared rapid gradient echo (MPRAGE) sequence was used to acquire high-resolution T1-weighted anatomical images (repetition time $=1,900 \mathrm{~ms}$, echo time $=$ $2.52 \mathrm{~ms}$, inversion time $=900 \mathrm{~ms}$, flip angle $=9$ degrees, resolution matrix $=256 \times 256$, slices $=176$, thickness $=$ $1.0 \mathrm{~mm}$, voxel size $=1 \times 1 \times 1 \mathrm{~mm}^{3}$ ).

\section{Preprocessing of structural data}

The MR images were processed using SPM8 (Wellcome Department of Cognitive Neurology, London, UK) implemented in MATLAB 7.8 (MathWorks Inc., Natick, MA, USA). First, each MRI image was displayed in SPM8 to filtrate gross anatomical abnormalities. For more accurate image registration, the reorientation of the images was manually 
fixed to the anterior commissure. Then, we used the new segmentation feature in SPM8 to segment the images into six tissues: gray matter, white matter, cerebrospinal fluid, the skull, soft tissue outside the brain, and air and other material outside of the head. Subsequently, we performed diffeomorphic anatomical registration through exponentiated Lie (DARTEL) algebra in SPM8 for registration, normalization, and modulation (Ashburner, 2007). The DARTEL registration involves first computing the specific template using the average tissue probability maps from all the participants, followed by warping each participant's segmented maps into the specific template. To improve the alignment and achieve a more accurate intersubject registration, the procedure was repeated until a best study-specific template was generated. To ensure conservation of regional differences in the absolute amounts of GM, the image intensity of each voxel was modulated by the Jacobian determinants. The registered images were transformed to Montreal Neurological Institute (MNI) space. Finally, the normalized modulated images (GM images) were smoothed with a 10-mm full-width-at-half maximum Gaussian kernel to increase the signal-to-noise ratio.

\section{Statistical analyses}

Statistical analyses of the brain imaging data were performed using SPM8. In the whole-brain analysis, multiple regression analysis was used to explore the association between GMV and individual differences in family SES. The family SES was used as the variable of interest. Total brain GMV, age, and sex were entered as covariates of no interest to control for the possible effects of these variables. In another whole-brain analysis, multiple regression analysis was used to explore the association between GMV and individual differences in parents' subjective SES rating by participants. The subjective parental SES was used as the variable of interest. Total brain GMV, age, and sex were entered as covariates of no interest to control for their possible effects. We also applied explicit masking using the population-specific masking toolbox in SPM8 to restrict the search volume to gray matter and white matter. This approach was used instead of absolute or relative threshold masking to reduce the risk of false negatives caused by overly restrictive masking, in which potentially interesting voxels are excluded from the statistical analysis (Ridgway et al., 2009). For all analyses, the cluster-level statistical threshold was set at $p<.05$ and corrected using nonstationary cluster correction (Hayasaka, Phan, Liberzon, Worsley, \& Nichols, 2004) with an underlying voxel level of $p<.001$.

\section{Mediation analysis}

To test whether the regional GMV could explain the relationship between young adults' depression-related traits and family SES, we performed a mediation analysis. A mediating variable $(M)$ is a variable that is part of the causal pathway by which an independent variable $(X)$ affects a dependent variable $(Y)$. Mediation analyzes were conducted using the indirect macro designed for SPSS (Preacher \& Hayes, 2008). In this study, $X$ is the family SES, $Y$ is the N3 measured by NEO-PI-R, and $M$ is the GMV of regions associated with family SES and family income, which were extracted from our own correct result. First, we saved our corrected result of the cluster of the significant cluster (mPFC extending to the ACC) correlated with the composite SES and income as ROI separately, then we extracted the two separate ROI signals (the volume of this ROI) from each participant using SPM8 toolbox. Age, sex, and the total GMV of individual brains were used as covariates in the model. This macro uses bootstrapped sampling to estimate the indirect mediation effect. In this analysis, 2,000 bootstrapped samples were drawn, and bias corrected $95 \%$ bootstrap confidence intervals (CI) were reported. CI that do not include zero indicate a significant indirect effect of the independent variable on the dependent variable through the mediators (Preacher \& Hayes, 2008).

\section{Results}

\section{Sample descriptive statistics}

The averaged standardized ( $z$ score) values of the two index variables educational qualification of subjects' parents and annual family income for each participant was used as an index of family SES. Higher $z$ scores indicated higher family SES. Table 1 lists the characteristics of demographics of the total sample. As indicated in Table 1, SES was negatively correlated with $\mathrm{N} 3(r=-.181, p=.004)$, indicating that the participants from higher SES families have lower rates of depression. When we use educational qualification of subjects' parents and annual family income as separate index of family SES, the result showed that family incomes were negatively correlated with $\mathrm{N} 3(r=-.178, p=.004)$, but subjects' parental educational qualifications were marginally significantly negatively correlated with $\mathrm{N} 3(r=-.120, p=.056)$. The results of this study may show that family income is more important in driving depression-related traits than are parental educational qualifications. In addition, the results showed that SES was positively correlated with General intelligence ( $r=$ $.223, p<.001)$, indicating that SES may be associated with General intelligence. Moreover, when we tested the relationship between SES and total brain volume and total gray volume, the results showed that SES had no significant correlation with total brain volume $(r=-.085, p=.178)$ and total gray volume ( $r=-.088, p=.163)$; when we also test the relationship between parental education and family income, the results showed that years of education correlated with family income positively $(r=.403, p<.001)$. In the present study, 
Table 1 The characteristics of demographic information about the large young sample $(n=253)$

\begin{tabular}{lcccc}
\hline Items & Mean & $S D$ & range & $\begin{array}{c}\text { Association } \\
\text { with family SES }^{\text {a }}\end{array}$ \\
\hline Age & 19.96 & 1.35 & $18-27$ & \\
Family income & 3.50 & 1.24 & $1-6$ & $-0.178^{* *}$ \\
with N3 & \\
Parental education years & 9.77 & 3.05 & $4.5-17$ & $1-9$ \\
Parental subjective social status & 5.13 & 1.56 & $-1.64-2.10$ & $0.260^{* *}$ \\
Family SES (z score) & -0.05 & 0.82 & $60-72$ & $0.223^{* *}$ \\
General intelligence & 66.23 & 3.06 & $11-39$ & $-0.181^{* *}$ \\
N3 (depression-related trait) & 22.19 & 5.05 & & $-0.181^{* *}$ \\
\hline
\end{tabular}

${ }^{\text {a }}$ Pearson bivariate correlations; shown are $r$ values

${ }^{*} p<.05 .{ }^{* *} p<.01$

the distribution of the N3 is a similarly normal distribution (see Fig. 1).

\section{Correlation between participants' GMV and their family SES}

After we entered age, sex, and global volumes of gray matter as covariates and family SES as an interest covariate into the regression model, a multiple regression analysis revealed that family SES was significantly and negatively correlated with GMV in a cluster that mainly included areas in the mPFC extending to the ACC $(x=0, y=49, z=14$, cluster size $=3$, 230 voxels, $t=-4.60, p<.05$, corrected; see Fig. $2 \mathrm{~A})$. We also examined the association between participants' rGMV and their family SES and tested whether these associations differed between sexes. The analysis of the interaction between sex and family SES on rGMV did not reveal any significant results.

To test whether the parental subjective social status rating by participants affected participants' brain structure, we entered age, sex, and global volumes of gray matter as covariates and parents' subjective social status rating by participants as an interest covariate into the regression model. A multiple regression analysis revealed that no brain cluster was significantly correlated with parents' subjective social status rating by participants. To control for parental subjective social status affect, in the multiple regression model we entered age, sex, global volumes of gray matter, and parents' subjective social status rating by participants as covariates and family SES as interest covariate into the regression model. The multiple regression analysis revealed that family SES was

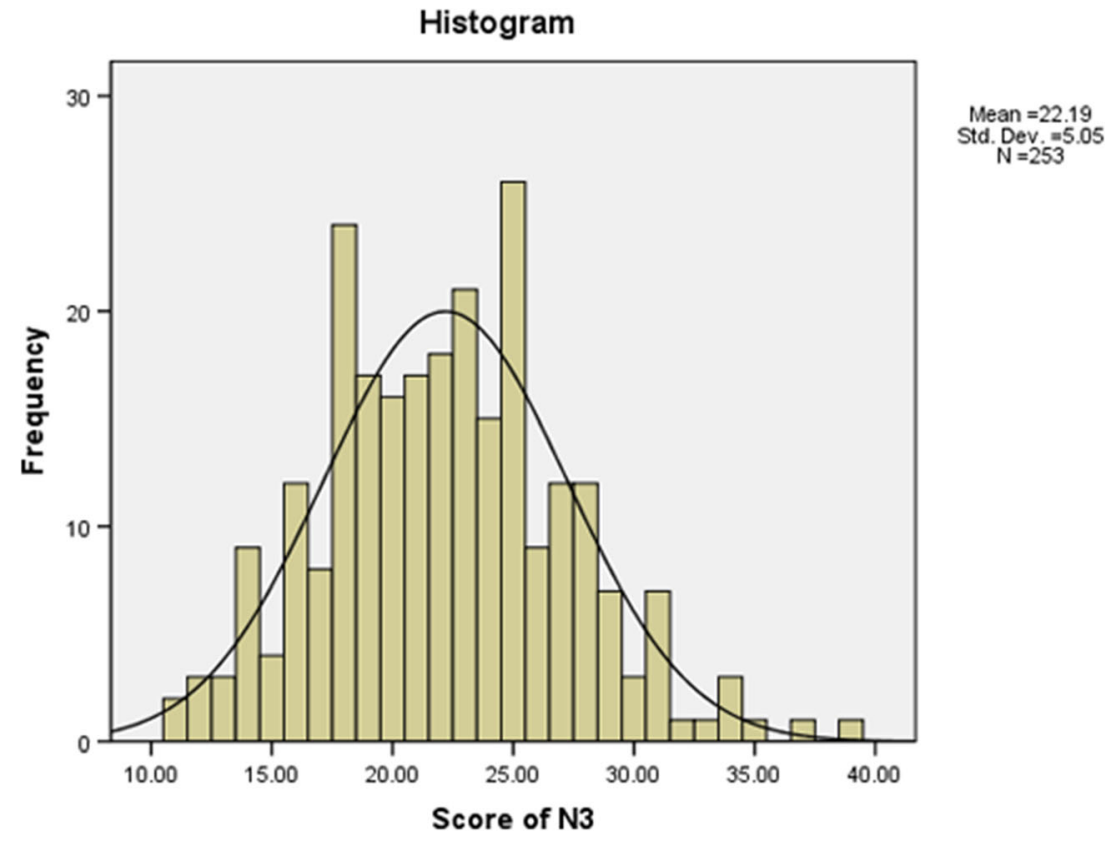

Fig. 1 The distribution of the N3 
A

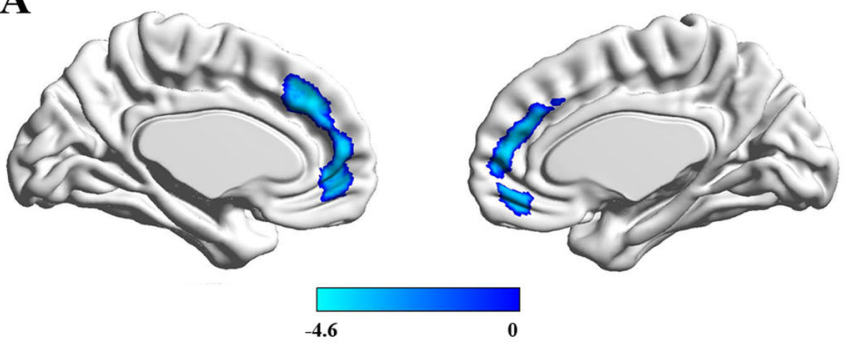

$\mathbf{L}$

B

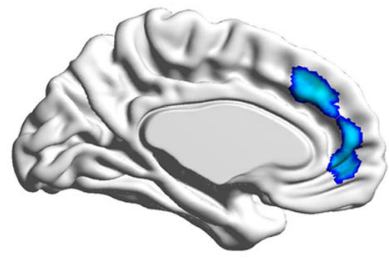

$\mathbf{R}$

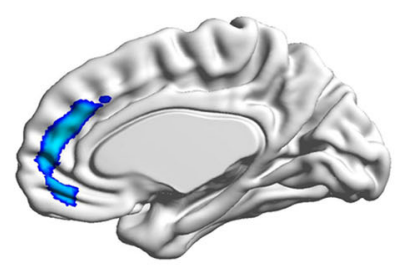

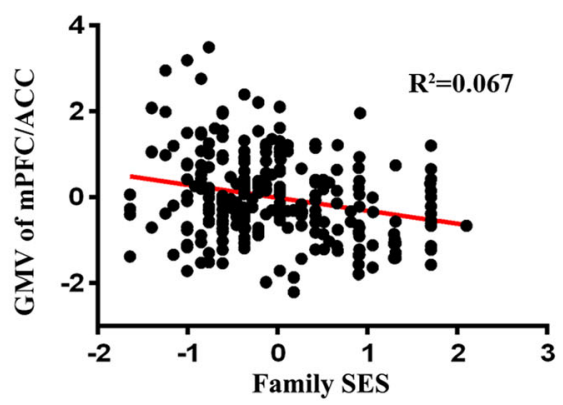

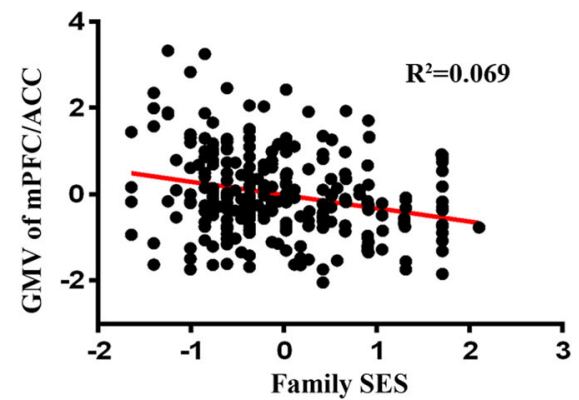

Fig. 2 A The regions of negative association between GMV and family SES (the results are $p<.05$, corrected) and the partial correlations (control age, sex, and whole brain gray volume) in a scatterplot between family SES and mean GMV within a significant cluster mainly included areas in the $\mathrm{mPFC}$ extending to the ACC. B The regions of negative association between GMV and family SES (the results are $p<.05$, corrected) and the

significantly and negatively correlated with GMV in a cluster that mainly included areas in the $\mathrm{mPFC}$ extending to the ACC $(x=2, y=48, z=15$, cluster size $=3$, 183 voxels, $t=-4.62 ; p<.05$, corrected; see Fig. 2B).

To test whether family income and parental education levels have different effects on the participants' brain structure, we entered age, sex, and global volumes of gray matter as covariates and family income and parental education levels as interest covariates into the regression model separately. The multiple regression analysis revealed that family incomes were significantly and negatively correlated with the GMV in a cluster that mainly included areas in the $\mathrm{mPFC}$ extending to the ACC $(x=2, y=46, z=17$, cluster size $=2,965$ voxels, $t$ $=-4.82 ; p<.05$, corrected; see Fig. 3A). There were no significant results between parental education and subjects' brain structures within the nonstationary corrections, but when we loosen the correct standards (set the $p$ value to .005 and cluster size to 500), the result showed that parental education levels were significantly and negative correlated with GMV in a cluster that mainly included areas in the mPFC extending to the ACC $(x=-6, y=36, z=40$, cluster size $=709$ voxels, $t=$ 3.8; see Fig. 3B). These results show that family income and parental education levels may have similar effects on participants' brain structure development.

The behavior results showed that family SES related to general intelligence. Thus, to test whether the subjects' partial correlations (control age, sex, parents' subjective social status, and whole brain gray volume) in a scatterplot between family SES and mean GMV within a significant cluster mainly included areas in the $\mathrm{mPFC}$ extending to the ACC after control for parental subjective social status affect

general intelligence affected the results of the above models, we entered age, sex, global volumes of gray matter, and participants' general intelligence as covariates and family SES, family income, and parental education levels as interest covariates into the regression model separately. The multiple regression analysis revealed that the significant clusters in these models did not change. The results show that family SES was significantly and negative correlated with the GMV in a cluster that mainly included areas in the mPFC extending to the $\operatorname{ACC}(x=2, y=48, z=15$, cluster size $=3,089$ voxels, $t=$ 4.60; $P<0.05$, corrected; see Fig. 4A), family income was significantly and negative correlated with the GMV in a cluster that mainly included areas in the mPFC extending to the ACC $(x=2, y=46, z=17$, cluster size $=2,844$ voxels, $t=$ $4.73 ; p<.05$, corrected; see Fig. 4B), and parental education levels were negatively correlated with the GMV in a cluster that mainly included areas in the mPFC extending to the ACC $(x=-6, y=36, z=40$, cluster size $=638$ voxels, $t=-3.6$; set the $p$ value to .005 and the cluster size to 500 , uncorrected; see Fig. 4C).

Previous studies suggested that SES was associated with the volume of the hippocampus and amygdala. To test whether in our samples the SES was associated with hippocampus and amygdala volumes, we extracted the hippocampal and amygdala volume regions based on the anatomy template (AAL) as regions of interest (ROIs). The results revealed no 
A

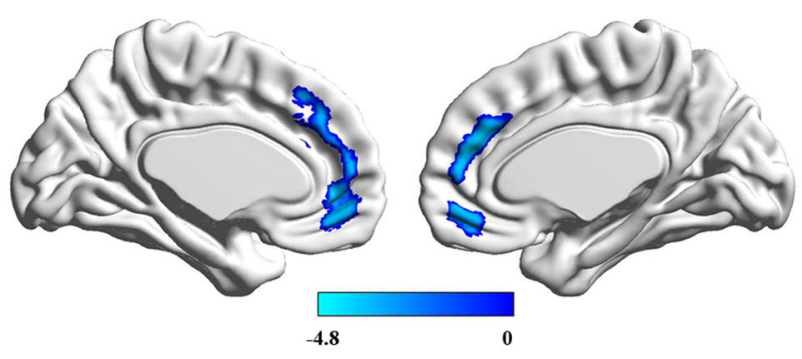

$\mathbf{L}$

B

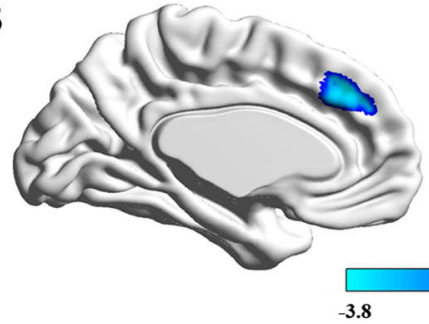

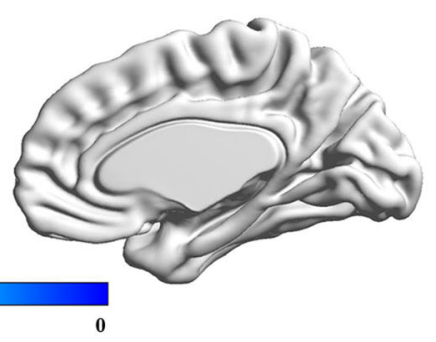

$\mathbf{R}$
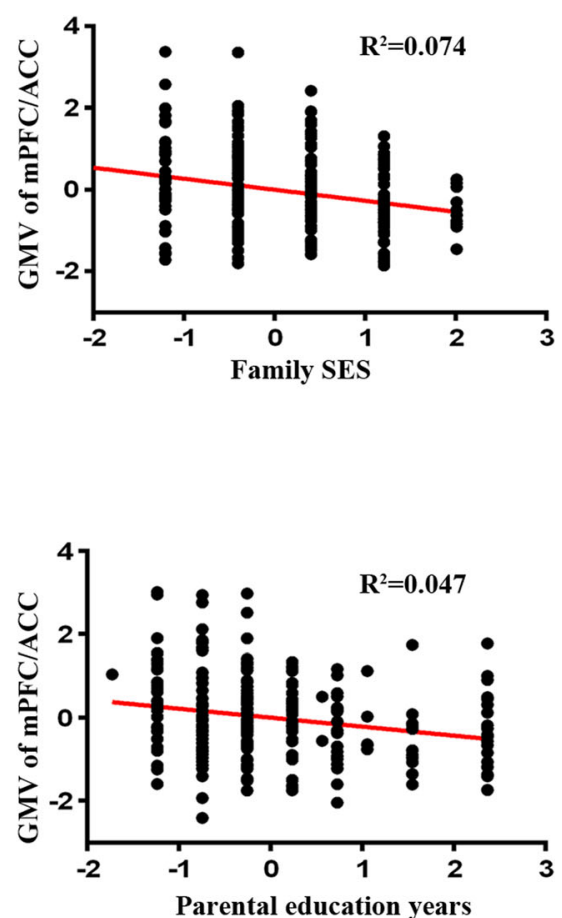

Fig. 3 A The regions of negative association between GMV and family income (the results are $p<.05$, corrected) and the partial correlations (control age, sex, and whole brain gray volume) in a scatterplot between family income and mean GMV within a significant cluster mainly included areas in the mPFC extending to the ACC. B The regions of negative association between GMV and parents' education (the results uncorrected for vision) and the partial correlations (control age, sex, and whole brain gray volume) in a scatterplot between parental education and mean GMV within a significant cluster mainly included areas in the $\mathrm{mPFC}$ extending to the ACC. significant correlation between family SES and hippocampal (for left hippocampal, $r=-.070, p=.267$; for right hippocampal, $r=-.065, p=.305$ ) or amygdala (for left amygdala, $r=$ $-.062, p=.326$; for right amygdala, $r=-.035, p=.578$ ) volume.

\section{Mediation results}

Indirect mediation effects can be interpreted as the strength of the relationship between family SES and participants' depression-related traits when accounting for mediating pathways. Depression-related traits (N3) were negatively associated with family SES $(r=-.181, p=.004)$. Family SES was negatively related to the volume of the mPFC extending to the ACC $(r=-.263, p<.001)$. N3 were positively related to the volume of the $\mathrm{mPFC}$ extending to the ACC $(r=.263, p<.001)$. To test the significance of the indirect effect between N3 and family SES, bootstrap resampling was used. The results showed a significant indirect effect (partial mediation effect) between family SES and N3, CI [$0.749,-0.179]$, through the volume of the mPFC extending to the ACC (see Fig. 5A). Depression-related traits (N3) were negatively associated with family income $(r=-.178, p=.004)$. Family income was negatively related to the volume of the mPFC extending to the ACC $(r=-.279, p<.001)$. N3 were positively related to the volume of $\mathrm{mPFC}$ extend to ACC $(r=$
$.205, p=.001)$. To test the significance of the indirect effect between N3 and family income, bootstrap resampling was used. The results showed a significant indirect effect (partially a mediation effect) between family income and N3, CI [$0.526,-0.075]$, through the volume of the mPFC extending to the ACC (see Fig. 5B).

\section{Discussion}

In this study, we investigated the neural basis of the relationship between depression-related traits and family SES in young healthy individuals. The behavioral results showed that family SES, especially family income, was related to young adults' depression-related traits. In addition, family SES was positive related to young adults' general intelligence; it is plausible that children from higher SES families have greater opportunities for and support in cognitive engagement and learning than children from more disadvantaged homes (Bradley \& Corwyn, 2002; Von Stumm \& Plomin, 2015). VBM results showed that family SES and family income were negatively correlated with the rGMV in the mPFC extending to the ACC. In addition, the mediation analysis showed that the volume of the mPFC extending to the ACC mediated the association between the family SES, especially family incomes, and the participants' depression-related traits. Taken 
$\mathbf{L}$

A

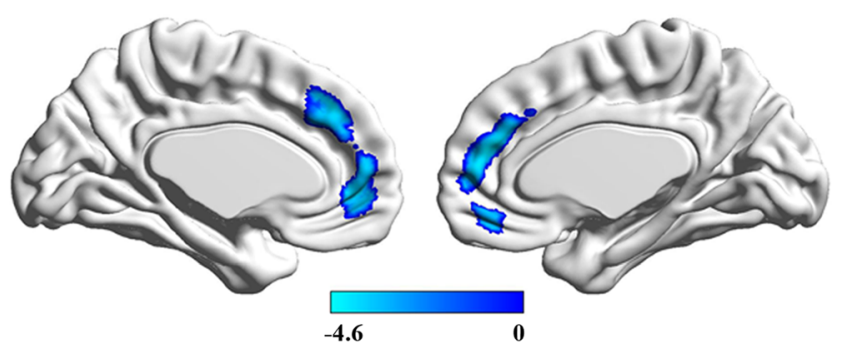

B

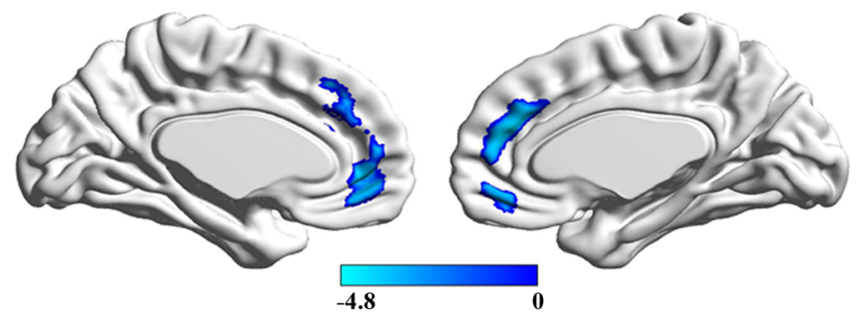

C

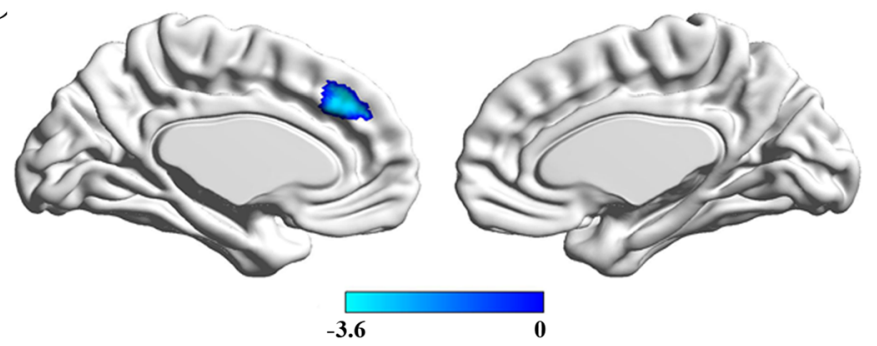

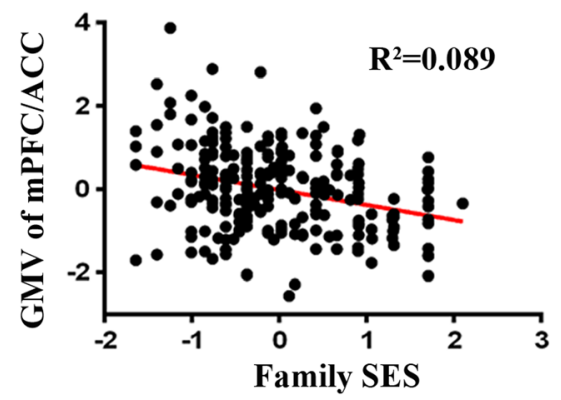
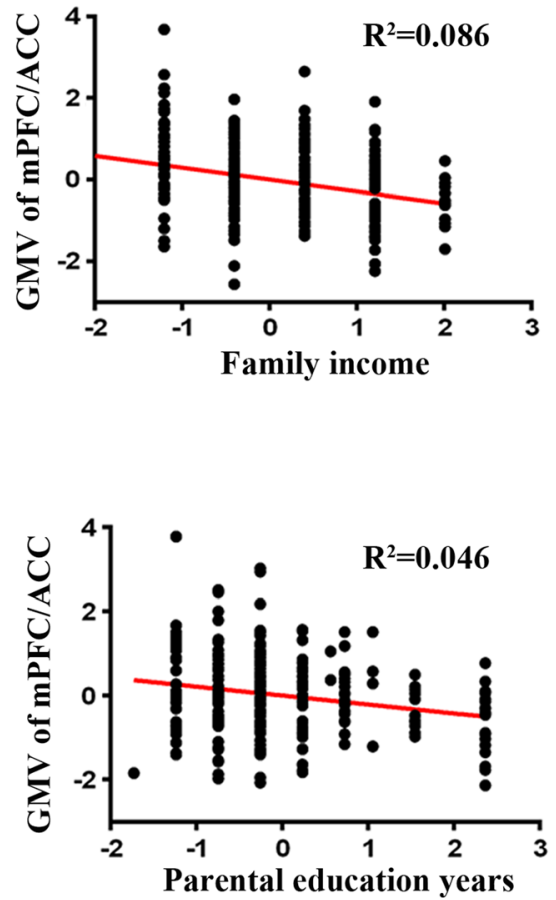

Fig. 4 A The regions of negative association between GMV and family SES (the results are $p<.05$, corrected) and the partial correlations (control age, sex, whole brain gray volume, and general intelligence) in a scatterplot between family income and mean GMV within a significant cluster mainly included areas in the mPFC extending to the ACC. B The regions of negative association between GMV and family income (the results are $p<.05$, corrected) and the partial correlations (control age, sex, whole brain gray volume, and general intelligence) in a scatterplot

together, our results suggest that the regions of the mPFC extending to the ACC may play an important role in the family SES, especially with regards to family income and depressionrelated traits. We think it is possible that in these brain regions, associations between parental education and young adults' brain gray matter volume area and personality may be mediated by the ability of more highly educated parents to earn higher incomes (Noble et al., 2015).

The association between family SES and rGMV in the $\mathrm{mPFC}$ extending to the ACC is consistent with previous studies that reported the role of the PFC extending to the ACC in SES. For instance, Tomarken and his colleagues found a between family income and mean GMV within a significant cluster mainly included areas in the mPFC extending to the ACC. C The regions of negative association between GMV and parental education (results uncorrected for vision) and the partial correlations (control age, sex, whole brain gray volume, and general intelligence) in a scatterplot between parents' education and mean GMV within a significant cluster mainly included areas in the mPFC extending to the ACC

relative left-frontal hypoactivity in lower SES adolescents (Tomarken et al., 2004). Furthermore, a recent study found that family SES significantly predicted cortical thickness in the right anterior cingulate gyrus and left superior frontal gyrus (Lawson et al., 2013). A growing body of evidence suggests that the mPFC plays an important role in emotional processing as well as the up-regulation and down-regulation of emotion (Kim \& Hamann, 2007). One important function of the $\mathrm{mPFC}$ is the top-down regulation of stress and threatrelated responding and coping processes mediated by subcortical limbic areas, including the hippocampus, amygdala, and hypothalamus (Compas, 2006; McEwen \& Gianaros, 2010). 


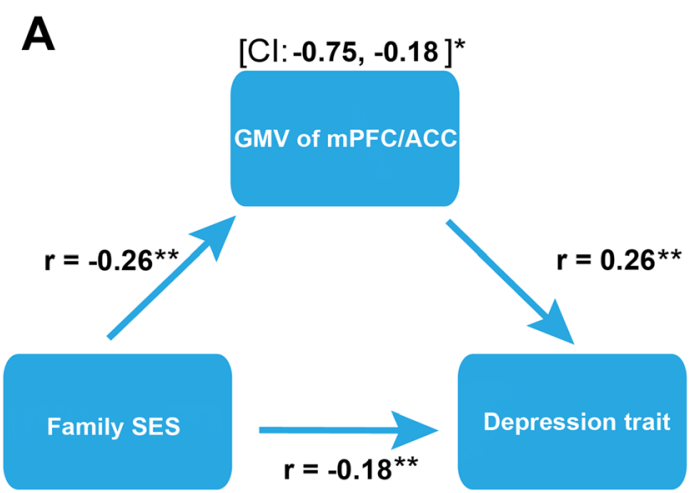

Fig. 5 A The mediation results and the results showed that the GMV of the mPFC extending to the ACC partly mediated the association between family SES and depression-related trait (N3). B The mediation results and

In addition, recent brain lesion and neuroimaging evidence indicate that the medial prefrontal cortex (mPFC) plays an important role in constraining the HPA axis under stressrelated conditions (Diorio, Viau, \& Meaney, 1993). Furthermore, recent reports have shown that volumetric changes in the $\mathrm{mPFC}$ are related to stress (Carballedo et al., 2012; Perez-Cruz, Simon, Czéh, Flügge, \& Fuchs, 2009; Sullivan, 2004).

Most of the work on the prefrontal cortex and stress-related processes in humans, particularly within the context of SES research, has focused on areas of the ACC (McEwen \& Gianaros, 2010). Some studies showed that ACC linked to stress-related processes (Lederbogen et al., 2011). These processes include the appraisal of salient environmental and personal events, the experience of emotional states, and the regulation of behavioral and autonomic responses to stressful stimuli (Bush et al., 2000; Paus, 2001; Vogt, 2005). Stress had both direct and indirect effects on mental health (Cohen, Doyle, \& Baum, 2006; Cohen et al., 1997; Vitaliano et al., 2002). In addition, a study indicated that the lower subjective social status is related to a smaller volume of the perigenual anterior cingulate cortex (Gianaros et al., 2007), a region that is involved in experiencing emotions and regulating behavioral and physiological reactivity to stress and affective disorders (Ochsner \& Gross, 2005; Ressler \& Mayberg, 2007). In contrast to a previous study by Brito and Noble (2014), which found that SES was positively correlated with the $\mathrm{mPFC} / \mathrm{ACC}$, the result of a negative correlation in the present study might be due to the differences in the sample, especially the age span, different analysis methods and culture. Taken together, these results may suggest that the volumetric changes in the mPFC/ACC associated with family SES may play an important role in emotional regulation.

The results showed that the relationship between family income and depression-related traits was partly mediated by the mPFC extending to ACC volume. Many studies have suggested that increased traits of depression or neuroticism are vulnerability factors for the development of depressive

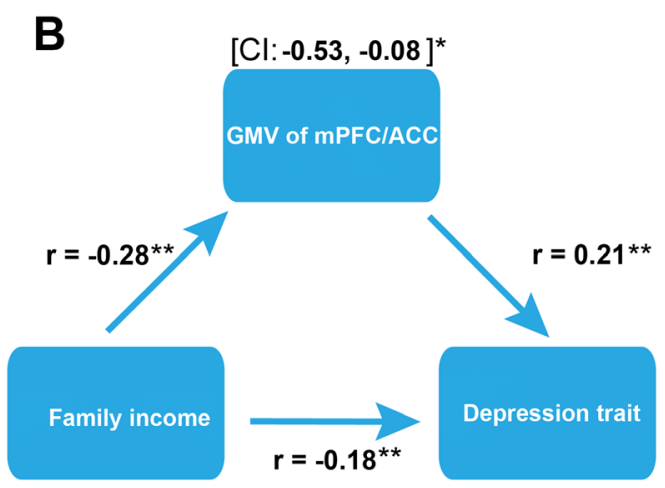

the results showed that the GMV of the mPFC extending to the ACC partly mediated the association between family income and depressionrelated trait $(\mathrm{N} 3)$

disorders (Boyce, Parker, Barnett, Cooney, \& Smith, 1991; Chioqueta \& Stiles, 2005). Depressive patients frequently have negative thoughts about themselves, and this tendency is associated with other negative thinking patterns and memory biases. In general, it serves to sustain negative emotional processing (Yoshimura et al., 2010). Moreover, some MRI studies have shown that key areas involved in emotional regulation, such as the cingulate cortex and prefrontal cortex (PFC) may exhibit functional and structural brain anomalies in depressed patients (Amico et al., 2011; Campbell, Marriott, Nahmias, \& MacQueen, 2004; S. C. Matthews, Strigo, Simmons, Yang, \& Paulus, 2008; Yoshimura et al., 2010). For example, imaging studies of self-referential encoding tasks indicate that the $\mathrm{MPFC}$ and the anterior cingulate cortex (ACC) are activated during the processing of emotional information (Fossati et al., 2003; Gusnard, Akbudak, Shulman, \& Raichle, 2001; Phan et al., 2004), and activation of the mPFC correlates with BDI scores when depressive patients are engaged in negative self-referential processing (Killgore \& Yurgelun-Todd, 2006). In addition, the mPFC and the ACC are part of a default mode network, and several studies have suggested that this default mode network is involved in emotional processing (D'Argembeau et al., 2005; Schneider et al., 2008). Furthermore, some researchers have found abnormal activations of the default mode network in depression during resting state and self-referential processing (Greicius, Supekar, Menon, \& Dougherty, 2009; Sheline et al., 2009). These results suggest that the activation in the $\mathrm{mPFC} / \mathrm{ACC}$ reflects the negative emotional processing that occurs in depression and that this processing is associated with depressive symptoms.

There are some limits in our findings. First, the sample of this study consisted of highly educated, normal young adults, who may have fewer symptoms of depression than are found in the general population. Therefore, it is unknown whether our results would be consistent with clinical samples. Second, VBM analysis is a more comprehensive measure that integrates changes in cortical folding and thickness. Other direct 
measures of the brain structure (such as cortical thickness, surface area or local gyrification index) should be used in further studies to better understand the neural bias of the relationship between family SES and depression (G.-X. Wei et al., 2013). Finally, the effect sizes of the results in the present study were small, which may be caused by the relative larger sample size and simple linear regression analysis method we used (Olejnik \& Algina, 2000), tut the relationship between the two variables may not be just a simple linear correlation.

In summary, the results of this study indicate that volumetric changes in the mPFC extending to the ACC could account in part for the dysregulated forms of emotional regulation among individuals who report lower family SES. In addition, the mediation results also indicate that the $\mathrm{mPFC}$ extending to the ACC possibly plays an important role in the relationship between family SES, especially family income and depression-related traits. However, future longitudinal studies in larger samples are required to confirm these results.

Acknowledgments This research was supported by the National Natural Science Foundation of China (31070900; 30800293; 30970892; 31170983), the Program for New Century Excellent Talents in University (2011) by the Ministry of Education, the Fundamental Research Funds for the Central Universities (SWU1209101), China Postdoctoral Science Foundation funded project (2012 M510098), the Research Funds for Southwest University (SWU09103), the Key Discipline Fund of National 211 Project (NSKD11007), the Fundamental Research Funds for the Central Universities (swu1209101), the Program for New Century Excellent Talents in University (2011) by the Ministry of Education, China Postdoctoral Science Foundation funded project (2012M510098), and the postgraduate Innovation Foundation of Science and Technology of Southwest University (kb2011002).

\section{References}

Adler, N. E., Boyce, T., Chesney, M. A., Cohen, S., Folkman, S., Kahn, R. L., \& Syme, S. L. (1994). Socioeconomic status and health: The challenge of the gradient. American Psychologist, 49(1), 15.

Adler, N. E., Epel, E. S., Castellazzo, G., \& Ickovics, J. R. (2000). Relationship of subjective and objective social status with psychological and physiological functioning: Preliminary data in healthy, White women. Health Psychology, 19(6), 586.

Amico, F., Meisenzahl, E., Koutsouleris, N., Reiser, M., Möller, H.-J., \& Frodl, T. (2011). Structural MRI correlates for vulnerability and resilience to major depressive disorder. Journal of Psychiatry \& Neuroscience: $J P N, 36(1), 15$.

Ashburner, J. (2007). A fast diffeomorphic image registration algorithm. NeuroImage, 38(1), 95-113.

Bienvenu, O. J., Samuels, J. F., Costa, P. T., Reti, I. M., Eaton, W. W., \& Nestadt, G. (2004). Anxiety and depressive disorders and the fivefactor model of personality: A higher- and lower-order personality trait investigation in a community sample. Depression and Anxiety, 20(2), 92-97.

Boyce, P., Parker, G., Barnett, B., Cooney, M., \& Smith, F. (1991). Personality as a vulnerability factor to depression. The British Journal of Psychiatry, 159(1), 106-114.
Bradley, R. H., \& Corwyn, R. F. (2002). Socioeconomic status and child development. Annual Review of Psychology, 53(1), 371-399.

Brito, N. H., \& Noble, K. G. (2014). Socioeconomic status and structural brain development. Frontiers in Neuroscience, 8, 276.

Brooks-Gunn, J., \& Duncan, G. J. (1997). The effects of poverty on children. The Future of Children, 55-71.

Bush, G., Luu, P., \& Posner, M. I. (2000). Cognitive and emotional influences in anterior cingulate cortex. Trends in Cognitive Sciences, 4(6), 215-222.

Campbell, S., Marriott, M., Nahmias, C., \& MacQueen, G. M. (2004). Lower hippocampal volume in patients suffering from depression: A meta-analysis. American Journal of Psychiatry, 161(4), 598-607.

Carballedo, A., Lisiecka, D., Fagan, A., Saleh, K., Ferguson, Y., Connolly, G., . . . Frodl, T. (2012). Early life adversity is associated with brain changes in subjects at family risk for depression. World Journal of Biological Psychiatry, 13(8), 569-578.

Cheng, H., \& Furnham, A. (2014). The associations between parental socio-economic conditions, childhood intelligence, adult personality traits, social status and mental well-being. Social Indicators Research, 117(2), 653-664.

Chioqueta, A. P., \& Stiles, T. C. (2005). Personality traits and the development of depression, hopelessness, and suicide ideation. Personality and Individual Differences, 38(6), 1283-1291.

Cohen, S., Doyle, W. J., \& Baum, A. (2006). Socioeconomic status is associated with stress hormones. Psychosomatic Medicine, 68(3), 414-420.

Cohen, S., Line, S., Manuck, S. B., Rabin, B. S., Heise, E. R., \& Kaplan, J. R. (1997). Chronic social stress, social status, and susceptibility to upper respiratory infections in nonhuman primates. Psychosomatic Medicine, 59(3), 213-221.

Compas, B. E. (2006). Psychobiological processes of stress and coping. Annals of the New York Academy of Sciences, 1094(1), 226-234.

Costa, P. T., \& McCrae, R. R. (1992a). Neo Personality InventoryRevised (NEO PI-R). Odessa, FL: Psychological Assessment Resources.

Costa, P. T., \& McCrae, R. R. (1992b). Neo PI-R professional manual. Odessa: Psychological Assessment Resources.

D'Argembeau, A., Collette, F., Van der Linden, M., Laureys, S., Del Fiore, G., Degueldre, C., . . . Salmon, E. (2005). Self-referential reflective activity and its relationship with rest: A PET study. NeuroImage, 25(2), 616-624.

Devinsky, O., Morrell, M. J., \& Vogt, B. A. (1995). Contributions of anterior cingulate cortex to behaviour (Review article). Brain, $118(1), 279-306$.

Diorio, D., Viau, V., \& Meaney, M. J. (1993). The role of the medial prefrontal cortex (cingulate gyrus) in the regulation of hypothalamic-pituitary-adrenal responses to stress. The Journal of Neuroscience, 13(9), 3839-3847.

Dubow, E. F., Huesmann, L. R., Boxer, P., Pulkkinen, L., \& Kokko, K. (2006). Middle childhood and adolescent contextual and personal predictors of adult educational and occupational outcomes: A mediational model in two countries. Developmental Psychology, 42(5), 937.

Fleming, S. M., Weil, R. S., Nagy, Z., Dolan, R. J., \& Rees, G. (2010). Relating introspective accuracy to individual differences in brain structure. Science, 329(5998), 1541-1543.

Flett, G. L., Hewitt, P. L., Endler, N. S., \& Bagby, R. M. (1995). Conceptualization and assessment of personality factors in depression. European Journal of Personality, 9(5), 309-350.

Fossati, P., Hevenor, S. J., Graham, S. J., Grady, C., Keightley, M. L., Craik, F., \& Mayberg, H. (2003). In search of the emotional self: An fMRI study using positive and negative emotional words. American Journal of Psychiatry, 160(11), 1938-1945.

Gianaros, P. J., Horenstein, J. A., Cohen, S., Matthews, K. A., Brown, S. M., Flory, J. D., . . . Hariri, A. R. (2007). Perigenual anterior 
cingulate morphology covaries with perceived social standing. Social Cognitive and Affective Neuroscience, 2(3), 161-173.

Gianaros, P. J., Horenstein, J. A., Hariri, A. R., Sheu, L. K., Manuck, S. B., Matthews, K. A., \& Cohen, S. (2008). Potential neural embedding of parental social standing. Social Cognitive and Affective Neuroscience, 3(2), 91-96.

Goodman, E. (1999). The role of socioeconomic status gradients in explaining differences in US adolescents' health. American Journal of Public Health, 89(10), 1522-1528.

Goodman, E., Slap, G. B., \& Huang, B. (2003). The public health impact of socioeconomic status on adolescent depression and obesity. American Journal of Public Health, 93(11), 1844-1850.

Greicius, M. D., Supekar, K., Menon, V., \& Dougherty, R. F. (2009). Resting-state functional connectivity reflects structural connectivity in the default mode network. Cerebral Cortex, 19(1), 72-78.

Gusnard, D. A., Akbudak, E., Shulman, G. L., \& Raichle, M. E. (2001). Medial prefrontal cortex and self-referential mental activity: Relation to a default mode of brain function. Proceedings of the National Academy of Sciences, 98(7), 4259-4264.

Hackman, D. A., Farah, M. J., \& Meaney, M. J. (2010). Socioeconomic status and the brain: Mechanistic insights from human and animal research. Nature Reviews Neuroscience, 11(9), 651-659.

Hanson, J. L., Chandra, A., Wolfe, B. L., \& Pollak, S. D. (2011). Association between income and the hippocampus. PLoS ONE, 6(5), e18712.

Hayasaka, S., Phan, K. L., Liberzon, I., Worsley, K. J., \& Nichols, T. E. (2004). Nonstationary cluster-size inference with random field and permutation methods. NeuroImage, 22(2), 676-687.

Huprich, S. K. (2000). Describing depressive personality anlogues and dysthymics on the NEO-Personality Inventory-Revised. Journal of Clinical Psychology, 56(12), 1521-1534.

Jaeggi, S. M., Buschkuehl, M., Jonides, J., \& Perrig, W. J. (2008). Improving fluid intelligence with training on working memory. Proceedings of the National Academy of Sciences, 105(19), 68296833.

Kanai, R., \& Rees, G. (2011). The structural basis of inter-individual differences in human behaviour and cognition. Nature Reviews Neuroscience, 12(4), 231-242.

Killgore, W. D., \& Yurgelun-Todd, D. A. (2006). Ventromedial prefrontal activity correlates with depressed mood in adolescent children. Neuroreport, 17(2), 167-171.

Kim, S., \& Hamann, S. (2007). Neural correlates of positive and negative emotion regulation. Journal of Cognitive Neuroscience, 19(5), 776798.

Lane, R. D., Reiman, E. M., Axelrod, B., Yun, L.-S., Holmes, A., \& Schwartz, G. E. (1998). Neural correlates of levels of emotional awareness: Evidence of an interaction between emotion and attention in the anterior cingulate cortex. Journal of Cognitive Neuroscience, 10(4), 525-535.

Lawson, G. M., Duda, J. T., Avants, B. B., Wu, J., \& Farah, M. J. (2013). Associations between children's socioeconomic status and prefrontal cortical thickness. Developmental Science, 16(5), 641-652.

Lederbogen, F., Kirsch, P., Haddad, L., Streit, F., Tost, H., Schuch, P., . . . Deuschle, M. (2011). City living and urban upbringing affect neural social stress processing in humans. Nature, 474(7352), 498-501.

Luby, J., Belden, A., Botteron, K., Marrus, N., Harms, M. P., Babb, C., . . . Barch, D. (2013). The effects of poverty on childhood brain development: The mediating effect of caregiving and stressful life events. JAMA Pediatrics, 167(12), 1135-1142.

Manuck, S. B., Bleil, M. E., Petersen, K. L., Flory, J. D., John Mann, J., Ferrell, R. E., \& Muldoon, M. F. (2005). The socio-economic status of communities predicts variation in brain serotonergic responsivity. Psychological Medicine, 35(04), 519-528.

Marioni, R. E., Davies, G., Hayward, C., Liewald, D., Kerr, S. M., Campbell, A., . . . Hocking, L. J. (2014). Molecular genetic contributions to socioeconomic status and intelligence. Intelligence, 44, 26-32.

Marmot, M. G., Kogevinas, M., \& Elston, M. A. (1987). Social/economic status and disease. Annual Review of Public Health, 8(1), 111-135.

Matthews, K. A., Flory, J. D., Muldoon, M. F., \& Manuck, S. B. (2000). Does socioeconomic status relate to central serotonergic responsivity in healthy adults? Psychosomatic Medicine, 62(2), 231-237.

Matthews, S. C., Strigo, I. A., Simmons, A. N., Yang, T. T., \& Paulus, M. P. (2008). Decreased functional coupling of the amygdala and supragenual cingulate is related to increased depression in unmedicated individuals with current major depressive disorder. Journal of Affective Disorders, 111(1), 13-20.

McCrae, R. R. (2011). Personality theories for the 21 st century. Teaching of Psychology, 38(3), 209-214.

McEwen, B. S., \& Gianaros, P. J. (2010). Central role of the brain in stress and adaptation: Links to socioeconomic status, health, and disease. Annals of the New York Academy of Sciences, 1186(1), 190-222.

McLoyd, V. C. (1997). The impact of poverty and low socioeconomic status on the socioemotional functioning of African-American children and adolescents: Mediating effects. In R. W. Taylor \& M. C. Wang (Eds.), Social and emotional adjustment and family relations in ethnic minority families (pp. 7-34). Mahwah, NJ: Erlbaum.

McLoyd, V. C. (1998). Socioeconomic disadvantage and child development. American Psychologist, 53(2), 185.

Ming, W. D. Q. (1989). Revision on the Combined Raven's Test for the Rural in China Psychological Science, 5(004).

Noble, K. G., Houston, S. M., Brito, N. H., Bartsch, H., Kan, E., Kuperman, J. M., . . . Libiger, O. (2015). Family income, parental education and brain structure in children and adolescents. Nature Neuroscience, 18, 773-778.

Noble, K. G., Houston, S. M., Kan, E., \& Sowell, E. R. (2012). Neural correlates of socioeconomic status in the developing human brain. Developmental Science, 15(4), 516-527.

Ochsner, K. N., \& Gross, J. J. (2005). The cognitive control of emotion. Trends in Cognitive Sciences, 9(5), 242-249.

Olejnik, S., \& Algina, J. (2000). Measures of effect size for comparative studies: Applications, interpretations, and limitations. Contemporary Educational Psychology, 25(3), 241-286.

Operario, D., Adler, N. E., \& Williams, D. R. (2004). Subjective social status: Reliability and predictive utility for global health. Psychology \& Health, 19(2), 237-246.

Otero, G. A. (1997). Poverty, cultural disadvantage and brain development: A study of pre-school children in Mexico. Electroencephalography and Clinical Neurophysiology, 102(6), 512-516.

Paus, T. (2001). Primate anterior cingulate cortex: Where motor control, drive and cognition interface. Nature Reviews Neuroscience, 2(6), $417-424$.

Perez-Cruz, C., Simon, M., Czéh, B., Flügge, G., \& Fuchs, E. (2009). Hemispheric differences in basilar dendrites and spines of pyramidal neurons in the rat prelimbic cortex: Activity- and stress-induced changes. European Journal of Neuroscience, 29(4), 738-747.

Phan, K. L., Taylor, S. F., Welsh, R. C., Ho, S.-H., Britton, J. C., \& Liberzon, I. (2004). Neural correlates of individual ratings of emotional salience: A trial-related fMRI study. NeuroImage, 21(2), 768 780 .

Posner, M. I. (1995). Attention in cognitive neuroscience: An overview. In M. S. Gazzaniga (Ed.), The cognitive neurosciences (pp. 615624). Cambridge, MA: The MIT Press.

Preacher, K. J., \& Hayes, A. F. (2008). Asymptotic and resampling strategies for assessing and comparing indirect effects in multiple mediator models. Behavior Research Methods, 40(3), 879-891.

Raizada, R. D., Richards, T. L., Meltzoff, A., \& Kuhl, P. K. (2008) Socioeconomic status predicts hemispheric specialisation of the left inferior frontal gyrus in young children. NeuroImage, 40(3), 13921401. 
Ressler, K. J., \& Mayberg, H. S. (2007). Targeting abnormal neural circuits in mood and anxiety disorders: From the laboratory to the clinic. Nature Neuroscience, 10(9), 1116-1124.

Ridgway, G. R., Omar, R., Ourselin, S., Hill, D. L., Warren, J. D., \& Fox, N. C. (2009). Issues with threshold masking in voxel-based morphometry of atrophied brains. NeuroImage, 44(1), 99-111.

Robert, S. A., \& House, J. S. (2000). Socioeconomic inequalities in health: Integrating individual-, community-, and societal-level theory and research. In G. L. Albrecht, R. Fitzpatrick, \& S. C. Scrimshaw (Eds.), Handbook of social studies in health and medicine (pp. 115-135). London, England: Sage.

Saklofske, D., Kelly, I., \& Janzen, B. (1995). Neuroticism, depression, and depression proneness. Personality and Individual Differences, 18(1), 27-31.

Schneider, F., Bermpohl, F., Heinzel, A., Rotte, M., Walter, M., Tempelmann, C., . . . Northoff, G. (2008). The resting brain and our self: Self-relatedness modulates resting state neural activity in cortical midline structures. Neuroscience, 157(1), 120-131.

Sen, S., Nesse, R. M., Stoltenberg, S. F., Li, S., Gleiberman, L., Chakravarti, A., . . . Burmeister, M. (2003). A BDNF coding variant is associated with the NEO personality inventory domain neuroticism, a risk factor for depression. Neuropsychopharmacology, 28, 397-401.

Sheline, Y. I., Barch, D. M., Price, J. L., Rundle, M. M., Vaishnavi, S. N., Snyder, A. Z., . . . Raichle, M. E. (2009). The default mode network and self-referential processes in depression. Proceedings of the National Academy of Sciences, 106(6), 1942-1947.

Sullivan, R. (2004). Hemispheric asymmetry in stress processing in rat prefrontal cortex and the role of mesocortical dopamine. Stress: The International Journal on the Biology of Stress, 7(2), 131-143.

Takeuchi, H., Taki, Y., Nouchi, R., Hashizume, H., Sassa, Y., Sekiguchi, A., ... Miyauchi, C. M. (2014). Anatomical correlates of quality of life: Evidence from voxel-based morphometry. Human Brain Mapping, 35(5), 1834-1846.

Takeuchi, H., Taki, Y., Sassa, Y., Hashizume, H., Sekiguchi, A., Fukushima, A., \& Kawashima, R. (2011). Working memory training using mental calculation impacts regional gray matter of the frontal and parietal regions [Research Support, Non-U.S. Gov't]. PLoS ONE, 6(8), e23175. doi:10.1371/journal.pone.0023175

Tang, C., Li, A., Huang, H., Cheng, X., Gao, Y., Chen, H., . . Zuo, Q. (2012). Effects of lead pollution in SY River on children's intelligence. Life Science Journal, 9(3), 458-464.

Terracciano, A., Tanaka, T., Sutin, A. R., Sanna, S., Deiana, B., Lai, S., . . . Ferrucci, L. (2010). Genome-wide association scan of trait depression. Biological psychiatry, 68(9), 811-817.
Tomarken, A. J., Dichter, G. S., Garber, J., \& Simien, C. (2004). Resting frontal brain activity: Inkages to maternal depression and socioeconomic status among adolescents. Biological Psychology, 67(1), $77-102$.

Tomassini, V., Jbabdi, S., Kincses, Z. T., Bosnell, R., Douaud, G., Pozzilli, C., . . Johansen-Berg, H. (2011). Structural and functional bases for individual differences in motor learning. Human Brain Mapping, 32(3), 494-508.

Tracy, M., Zimmerman, F. J., Galea, S., McCauley, E., \& Stoep, A. V. (2008). What explains the relation between family poverty and childhood depressive symptoms? Journal of Psychiatric Research, 42(14), 1163-1175.

Vitaliano, P. P., Scanlan, J. M., Zhang, J., Savage, M. V., Hirsch, I. B., \& Siegler, I. C. (2002). A path model of chronic stress, the metabolic syndrome, and coronary heart disease. Psychosomatic Medicine, 64(3), 418-435.

Vogt, B. A. (2005). Pain and emotion interactions in subregions of the cingulate gyrus. Nature Reviews Neuroscience, 6(7), 533-544.

Von Stumm, S., \& Plomin, R. (2015). Socioeconomic status and the growth of intelligence from infancy through adolescence. Intelligence, 48, 30-36.

Vossen, H. G., van Os, J., Hermens, H., \& Lousberg, R. (2006). Evidence that trait-anxiety and trait-depression differentially moderate cortical processing of pain. The Clinical Journal of Pain, 22(8), 725-729.

Washburn, D. A., Smith, J. D., \& Taglialatela, L. A. (2005). Individual differences in metacognitive responsiveness: Cognitive and personality correlates. The Journal of General Psychology, 132(4), 446461.

Wei, D., Du, X., Li, W., Chen, Q., Li, H., Hao, X., . . Qiu, J. (2014). Regional gray matter volume and anxiety-related traits interact to predict somatic complaints in a non-clinical sample. Social Cognitive and Affective Neuroscience, 10(1), 122-128.

Wei, G.-X., Xu, T., Fan, F.-M., Dong, H.-M., Jiang, L.-L., Li, H.-J., . . . Zuo, X.-N. (2013). Can taichi reshape the brain? A brain morphometry study. PLOS ONE, 8(4), e61038.

Yoshimura, S., Okamoto, Y., Onoda, K., Matsunaga, M., Ueda, K., \& Suzuki, S.-I. (2010). Rostral anterior cingulate cortex activity mediates the relationship between the depressive symptoms and the medial prefrontal cortex activity. Journal of Affective Disorders, 122(1), 76-85.

Zimmerman, F. J., \& Katon, W. (2005). Socioeconomic status, depression disparities, and financial strain: What lies behind the incomedepression relationship? Health Economics, 14(12), 1197-1215. 